\title{
Measuring chiral imbalance with collisional energy loss
}

\author{
Stefano Carignano $\odot^{*}$ and Cristina Manuel $\odot^{\dagger}$ \\ Instituto de Ciencias del Espacio (ICE, CSIC) C. Can Magrans s.n., \\ 08193 Cerdanyola del Vallès, Catalonia, Spain \\ and Institut d'Estudis Espacials de Catalunya (IEEC) \\ C. Gran Capità 2-4, Ed. Nexus, 08034 Barcelona, Spain
}

(Received 10 March 2021; accepted 2 May 2021; published 2 June 2021)

\begin{abstract}
We compute the collisional energy loss of an energetic massive fermion crossing a chiral plasma at finite temperature characterized by an imbalance between the populations of left-handed and right-handed fermions. We find a new contribution to the energy loss which is proportional to the helicity of the test fermion and depends on the amount of chiral imbalance in the plasma. We then compute the difference between the energy loss of a fermion with the two opposite helicities, to assess whether this could be used to quantify the chiral imbalance in the plasma. We find that the leading contribution to these helicitydependent energy loss contributions comes from the exchange of hard photons (or gluons for QCD) with the medium constituents, and in some scenarios can become comparable to the leading-order result for a plasma without any chiral imbalance. We also evaluate the contribution arising from soft photon exchange, which is a subleading effect, and requires regularization. We illustrate how dimensional regularization is a well suited prescription to be applied to these energy loss computations.
\end{abstract}

DOI: 10.1103/PhysRevD.103.116002

\section{INTRODUCTION}

The measurement of the energy loss for a jet propagating through a high-temperature plasma is one of the most prominent quantities which can be used to characterize the properties of matter in scenarios such as heavy-ion collision experiments (see e.g., [1-4] for recent reviews). Of particular interest in such a context is the energy loss of a heavy parton produced in the early stages of the collision, which crosses the quark-gluon plasma interacting with the medium constituents.

Recently, it has been proposed that the quark-gluon plasma created in a heavy-ion collision could exhibit an imbalance between populations of left-handed and righthanded fermions, giving rise to several new macroscopic phenomena (see [5-7] for reviews).

Aside from such anomalous transport phenomena, the presence of a chiral imbalanced system should affect the interaction of an energetic fermion with the medium, and in particular its collisional energy loss due to interaction with the constituents of the plasma. This feature has already appeared in a calculation of the damping rate of a massless

\footnotetext{
carignano@ice.cat

†cmanuel@ice.csic.es
}

Published by the American Physical Society under the terms of the Creative Commons Attribution 4.0 International license. Further distribution of this work must maintain attribution to the author(s) and the published article's title, journal citation, and DOI. Funded by SCOAP . fermion in an imbalanced dense system at zero temperature [8], where it was found that the interaction with the medium particles mediated by soft photons distinguishes between different photon circular polarizations and depends on the chirality of the test particle. This in turn suggests to us that a closer investigation of the energy loss might even help shed some light on the amount of chiral imbalance originated in a heavy-ion collision experiment.

In order to investigate this aspect in more detail, in this work we compute the collisional energy loss of an energetic massive fermion traversing a chiral plasma at finite temperature $T$ made of unequal populations of left-handed and right-handed massless fermions. We consider the fermion energy $E$ to be much larger than the temperature, $E \gg T$. The imbalance is parametrized by the chiral chemical potential $\mu_{5}=\mu_{R}-\mu_{L}$, where $\mu_{R / L}$ refer to the chemical potential associated with right- and left-handed fermions of the medium, respectively. We start by considering an electromagnetic plasma, and later on we discuss how to generalize our main results to QCD.

It is worth recalling that helicity is not a Lorentz invariant quantity. Our computation is carried out in the frame at rest with the plasma, but it should be possible to generalize it to a more convenient lab frame.

The calculation of the collisional energy loss involves considering contributions for both hard and soft momenta of the exchanged photon (recall that hard and soft refer to scales of order $T$ and $e T$, respectively, where $e$ is the gauge coupling constant, and that the soft scales require the resummation of hard thermal loops $[9,10])$. For the leading 
contribution in a plasma without chiral imbalance, both contributions when taken separately exhibit divergencies which cancel when adding them up. This separation of scales is typically performed using a sharp momentum cutoff, a procedure which in principle spoils gauge invariance. In this work, in order to calculate the new contributions arising in a chiral imbalanced plasma we will follow the same philosophy, but employing dimensional regularization (DR) to regularize all intermediate results. As an illustrative example, in the Appendix A we repeat the known calculation of the leading contribution to the energy loss for a plasma without chiral imbalance employing DR. We stress that DR is a perfectly suited regularization method in the presence of powerlike divergences, as those we find in our computations.

We find that the leading contribution to the helicitydependent energy loss arises from the exchange of hard photons with the medium constituents, and it does not exhibit any sort of divergence. We also find a contribution to the helicity-dependent energy loss as arising from the exchange of soft photons, which however turns out to be perturbatively suppressed as compared to the hard contribution.

Let us finally mention that in a recent publication the collisional energy loss in a chiral medium has been considered, by studying the electromagnetic fields created by a moving electrical charge in the presence of the chiral magnetic current [11,12]. The effective field equations considered in that manuscript however neglect thermal effects, and only describe the physics of chiral plasmas in the static limit, as can be shown by evaluating the polarization tensors of the chiral plasma used in our manuscript.

This paper is structured as follows: in Sec. II we compute the leading hard contribution to the helicity-dependent collision rate, while in Sec. III we compute the leading soft one. We discuss our results in Sec. IV. In Appendix A we repeat the known calculation of the collision rate in a regular QED plasma without chiral imbalance, employing DR, the soft sector is considered in Appendix A 1, while Appendix A 2 considers the hard sector. We provide in App. B some of the DR integrals that we used in our computations.

We work with natural units $\hbar=c=1$ and metric $g^{\mu \nu}=\operatorname{diag}(1,-1,-1,-1)$. We denote four-momenta with capital letters, $K^{\mu}=\left(k^{0}, \mathbf{k}\right)$, and the modulus of threemomenta as $k=|\mathbf{k}|$.

\section{HARD CONTRIBUTION TO THE COLLISIONAL ENERGY LOSS OF A MASSIVE FERMION IN THE CHIRAL PLASMA}

Let us start by computing the damping rate of a massive particle with mass $M$, momentum $\mathbf{p}=\mathbf{v} E$, energy $E=$ $\sqrt{p^{2}+M^{2}}$ and a given helicity $\lambda$, due to collision with massless fermions in the chiral plasma, characterized by a temperature $T$ and chiral chemical potential $\mu_{5}$.

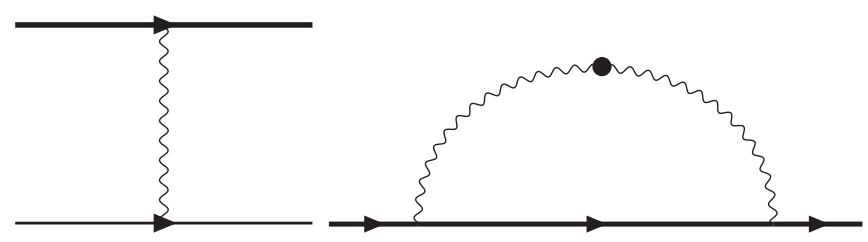

FIG. 1. Feynman diagrams for the energy loss calculation. Left: scattering of a heavy fermion (thick line) with a medium fermion via a photon exchange. Right: heavy fermion self-energy. The dot denotes a resummed medium photon.

The relevant scattering diagram is given by Fig. 1 left, depicting the scattering of a heavy fermion of a given helicity $\lambda$ with a massless one in the medium with chirality $\chi$ via photon exchange.

The corresponding matrix element squared is given by

$$
\begin{aligned}
|\mathcal{M}|_{(\lambda \chi)}^{2}= & e^{4} D_{\mu \nu}(Q) D_{\alpha \beta}^{\dagger}(Q) \\
& \times \operatorname{Tr}\left[\mathcal{P}_{(\lambda, \mathbf{p})}(\not P+M) \gamma^{\mu}\left(\not P^{\prime}+M\right) \gamma^{\alpha}\right] \\
& \times \operatorname{Tr}\left[P_{(\chi)} K^{\prime} \gamma^{\nu} K \gamma^{\beta}\right],
\end{aligned}
$$

where $D_{\mu \nu}$ is the photon propagator, written in terms of energy and momentum transfer of the collision $Q^{\mu}=(\omega, \mathbf{q})$, which is assumed to be hard,

$$
\mathcal{P}_{(\lambda, \mathbf{p})}=\frac{1}{2}\left(1+\lambda \gamma^{5} \gamma^{0} \vec{\gamma} \cdot \hat{\mathbf{p}}\right), \quad \lambda= \pm,
$$

is the helicity projector and

$$
P_{(\chi)}=\frac{1+\chi \gamma_{5}}{2}, \quad \chi= \pm
$$

is the chirality projector. Note that for massless fermions, helicity and chirality agree, but this is not so otherwise.

The first trace, which depends on the helicity of the massive fermion, is given by

$$
\begin{aligned}
\operatorname{Tr} & {\left[\mathcal{P}_{(\lambda, \mathbf{p})}(\not \boldsymbol{P}+M) \gamma^{\mu}(\boldsymbol{K}+M) \gamma^{\nu}\right] } \\
= & 2\left\{\left[P^{\mu} K^{\nu}+P^{\nu} K^{\mu}+\left(M^{2}-P \cdot K\right) g^{\mu \nu}\right]\right. \\
& \left.-i \lambda \hat{p}^{i}\left[K_{\beta}\left(p^{i} \epsilon^{0 \mu \beta \nu}-p^{0} \epsilon^{i \mu \beta \nu}\right)+M^{2} \epsilon^{0 i \mu \nu}\right]\right\},
\end{aligned}
$$

while the second one, related to the chiral fermion in the medium, is given by

$$
\begin{aligned}
\operatorname{Tr}\left[P_{(\chi)} K^{\prime} \gamma^{\nu} K \gamma^{\beta}\right]= & 2\left\{\left[K^{\prime \nu} K^{\beta}+K^{\prime \beta} K^{\nu}-\left(K^{\prime} \cdot K\right) g^{\nu \beta}\right]\right. \\
& \left.+i \chi K_{\eta} K_{\rho}^{\prime} \epsilon^{\nu \beta \eta \rho}\right\} .
\end{aligned}
$$

Splitting the traces into symmetric and antisymmetric pieces, their product will give two contributions: 


$$
\begin{aligned}
|\mathcal{M}|_{(\lambda \chi)}^{2}= & 4 e^{4} D_{\mu \nu}(Q) D_{\alpha \beta}^{\dagger}(Q)\left\{\left[P^{\prime \mu} P^{\alpha}+P^{\prime \alpha} P^{\mu}+\left(M^{2}-P^{\prime} \cdot P\right) g^{\mu \alpha}\right]\left[K^{\prime \nu} K^{\beta}+K^{\prime \beta} K^{\nu}-\left(K^{\prime} \cdot K\right) g^{\nu \beta}\right]\right. \\
& \left.-\lambda \chi K_{\eta} K_{\rho}^{\prime} \epsilon^{\nu \beta \eta \rho} \hat{p}^{i}\left[P_{\sigma}^{\prime} p^{i} \epsilon^{0 \mu \sigma \alpha}-P_{\sigma}^{\prime} p^{0} \epsilon^{i \mu \sigma \alpha}+M^{2} \epsilon^{0 i \mu \alpha}\right]\right\} .
\end{aligned}
$$

The first contribution gives the usual result known from the literature $[9,10]$, whereas the second one depends both on the helicity of the heavy fermion as well as the chirality of the medium fermion. In a plasma where there is the same population of left- and right-handed fermions this latter contribution vanishes in the final computation of the damping rate or energy loss when summing over chiralities, but this is not the case otherwise.

The damping rate is given by integrating the matrix element squared over phase space and summing over the chiralities of the fermions in the plasma. We shall concentrate in the following in the interaction rate, which is related to the damping as $\Gamma_{\lambda}=2 \gamma_{\lambda}$. At leading order in the QED coupling constant we get

$$
\begin{aligned}
\Gamma_{\lambda}^{\text {hard }}= & \frac{1}{E} \int \frac{d^{3} p^{\prime}}{(2 \pi)^{3}} \frac{1}{2 E^{\prime}} \int \frac{d^{3} k}{(2 \pi)^{3}} \sum_{\chi= \pm} \frac{n_{\chi}(k)}{2 k} \int \frac{d^{3} k^{\prime}}{(2 \pi)^{3}} \frac{1-n_{\chi}\left(k^{\prime}\right)}{2 k^{\prime}}(2 \pi)^{4} \delta^{4}\left(P+K-P^{\prime}-K^{\prime}\right)|\mathcal{M}|_{(\lambda \chi)}^{2} \\
= & \frac{8 e^{4}}{E} \int \frac{d^{3} p^{\prime}}{(2 \pi)^{3}} \frac{1}{2 E^{\prime}} \int \frac{d^{3} k}{(2 \pi)^{3}} \sum_{\chi= \pm} \frac{n_{\chi}(k)}{2 k} \int \frac{d^{3} k^{\prime}}{(2 \pi)^{3}} \frac{1-n_{\chi}\left(k^{\prime}\right)}{2 k^{\prime}}(2 \pi)^{4} \delta^{4}\left(P+K-P^{\prime}-K^{\prime}\right) \\
& \times \frac{E^{2}}{Q^{4}}\left\{\left[2(k-\mathbf{v} \cdot \mathbf{k})\left(k^{\prime}-\mathbf{v}^{\prime} \cdot \mathbf{k}^{\prime}\right)+\frac{Q^{4}}{4 E^{2}}+\frac{M^{2} Q^{2}}{2 E^{2}}\right]+\lambda \chi\left(-\frac{Q^{2}}{2 E}\right)\left[v\left(k+k^{\prime}\right)-\hat{\mathbf{v}} \cdot\left(\mathbf{k}+\mathbf{k}^{\prime}\right)\right]\right\},
\end{aligned}
$$

where

$$
n_{\chi}(k)=\frac{1}{\exp \left(\left(k-\chi \mu_{5}\right) / T\right)+1}, \quad \chi= \pm
$$

is the occupation number for a fermion of chirality $\chi$. Thus, we assume that in the plasma there is only a chiral chemical potential and no baryonic chemical potential.

In the last row of Eq. (7) one can recognize the result for a symmetric plasma $[9,10]$, followed by the first nonvanishing correction in a chiral plasma, which is proportional to the product $\lambda \chi$ of the helicity and the chirality of the heavy and the light medium fermion, respectively. Note that this correction is $1 / E$ suppressed compared to the leading result.

Let us now consider a very energetic fermion $(E \gg T)$, and move to the energy loss $-d E / d x$, which can be obtained by multiplying the integrand of the damping rate by a factor $\left(E-E^{\prime}\right) / v[9]$.

If one simply takes the hard contribution computed in this section, the energy loss at leading order turns out to be infrared divergent. This is cured by appropriately taking into account the contribution from soft momenta in the exchanged photon, which requires a proper resummation. In $[9,10]$, this is implemented by introducing a cutoff which separates the hard and soft contributions of the computation. Even though the final result does not depend on the cutoff parameter, this regularization method has some clear drawbacks, including an ambiguity in the choice of the cutoff itself (as a matter of fact, two different ways have been employed in $[9,10])$. An alternative approach would be to employ dimensional regularization, which has several advantages over the cutoff regularization, as it preserves the gauge invariance. We illustrate the method in Appendix A.
On the other hand, the new contribution we compute in the following, which is proportional to the helicity of the external fermion, turns out to be finite and does not require any regularization. In order to focus on the new chirality and helicity-dependent effects we are computing, let us consider the difference between the energy loss associated with the two opposite helicities:

$$
\begin{aligned}
& \left.\Delta^{\text {hard }} \equiv\left(-\frac{d E_{\lambda=+}}{d x}\right)\right|_{\text {hard }}-\left.\left(-\frac{d E_{\lambda=-}}{d x}\right)\right|_{\text {hard }} \cdot \\
\Delta^{\text {hard }}= & \frac{16 e^{4}}{v E} \int \frac{d^{3} p^{\prime}}{(2 \pi)^{3}} \frac{1}{2 E^{\prime}} \int \frac{d^{3} k}{(2 \pi)^{3}} \sum_{\chi= \pm} \chi \frac{n_{\chi}(k)}{2 k} \\
& \times \int \frac{d^{3} k^{\prime}}{(2 \pi)^{3}} \frac{1-n_{\chi}\left(k^{\prime}\right)}{2 k^{\prime}}(2 \pi)^{4} \delta^{4}\left(P+K-P^{\prime}-K^{\prime}\right) \\
& \times\left(E-E^{\prime}\right) \frac{E^{2}}{Q^{4}}\left\{\left(-\frac{Q^{2}}{2 E}\right)\left[v\left(k+k^{\prime}\right)-\hat{\mathbf{v}} \cdot\left(\mathbf{k}+\mathbf{k}^{\prime}\right)\right]\right\} .
\end{aligned}
$$

At this point one can proceed and eliminate the $p^{\prime}$ integral with the $d=3$ spatial delta functions. The remaining delta of energy conservation can be approximated, again to leading order in a $1 / E$ expansion, as $\delta\left(\omega-\mathbf{v} \cdot \mathbf{q}-Q^{2} /\right.$ $(2 E)$ ), and neglecting higher order terms we can drop the $n_{F}\left(k^{\prime}\right)$ above due to the symmetries of the integrand [the expression in the second row of Eq. (10) is antisymmetric under the exchange $\mathbf{k} \leftrightarrow \mathbf{k}^{\prime}$ ].

By further introducing

$$
1=\int d^{d} q \delta^{(d)}\left(\mathbf{q}+\mathbf{k}-\mathbf{k}^{\prime}\right) \int d \omega \delta\left(\omega+k-k^{\prime}\right),
$$


we can eliminate the integral in $k^{\prime}$ and write it in terms of $q$, the momentum transfer.

At this point, one can use the delta function to carry out one of the angular integrals, and perform also all the trivial angular integrations. More specifically, we write $\delta(\omega+k-|\mathbf{k}+\mathbf{q}|)=2|\mathbf{k}+\mathbf{q}| \delta\left(Q^{2}+2 k \omega-2 \mathbf{k} \cdot \mathbf{q}\right)$ and use it to replace the $\mathbf{k} \cdot \mathbf{q}$ pieces in our expression. Performing an angle average over the directions of the incoming fermion and keeping only the leading $1 / E$ contribution we arrive at

$\Delta^{\mathrm{hard}}=-\frac{e^{4}}{4 \pi^{3} v^{3} E} \int_{0}^{\infty} d k\left[n_{+}(k)-n_{-}(k)\right] \int_{0}^{\infty} d q \int_{\omega_{-}}^{\omega_{+}} d \omega \frac{\omega}{Q^{2}}\left[\left(v^{2}-\frac{\omega^{2}}{q^{2}}\right)(2 k+\omega)\right] \Theta(|q-k| \leq|\omega+k| \leq q+k)$,

where $\Theta$ is the step function, and with $\omega_{ \pm}=E-\sqrt{E^{2}+q^{2} \mp 2 E v q}$. As in [10], we can again split the integrals in two regions,

$$
\Delta^{\text {hard }}=-\frac{e^{4}}{4 \pi^{3} v^{3} E}\left[\mathcal{I}_{1}+\mathcal{I}_{2}\right]
$$

The first integral is given by

$$
\begin{aligned}
\mathcal{I}_{1} & =\int_{0}^{\infty} d k\left[n_{+}(k)-n_{-}(k)\right] \int_{0}^{\frac{2 k}{1+v}} d q \int_{-v q}^{v q} d \omega \frac{\omega}{Q^{2}}\left[\left(v^{2}-\frac{\omega^{2}}{q^{2}}\right)(2 k+\omega)\right] \\
& =-\frac{2}{(1+v)^{2}} \frac{2}{3}\left[v\left(2 v^{3}-3\right)-\frac{3}{2}\left(v^{2}-1\right) \log \left(\frac{1+v}{1-v}\right)\right] \frac{\mu_{5}}{3}\left(\mu_{5}^{2}+\pi^{2} T^{2}\right) .
\end{aligned}
$$

The second contribution, which amounts to

$$
\mathcal{I}_{2}=\int_{0}^{\infty} d k\left[n_{+}(k)-n_{-}(k)\right] \int_{\frac{2 k}{1+v}}^{q_{\max }} d q \int_{q-2 k}^{\omega_{+}} d \omega \frac{\omega}{Q^{2}}\left[\left(v^{2}-\frac{\omega^{2}}{q^{2}}\right)(2 k+\omega)\right],
$$

with $q_{\max }=2 k(E+k) /(E(1-v)+2 k)$, needs some more careful treatment.

Following again $[9,10]$, we can treat separately the regime $E \ll M^{2} / T$, where we can approximate $\omega_{+} \simeq v q$, $q_{\max } \simeq 2 k /(1-v)$, obtaining

$\mathcal{I}_{2}^{\left(E \ll M^{2} / T\right)}=-\frac{1}{6(1+v)^{2}}\left[\frac{2 v}{v-1}\left(-21+3 v+23 v^{2}+7 v^{3}\right)+3(v-1)\left(7+13 v+9 v^{2}+3 v^{3}\right) \log \frac{1+v}{1-v}\right] \frac{\mu_{5}}{3}\left(\mu_{5}^{2}+\pi^{2} T^{2}\right)$,

whereas for $E \gg M^{2} / T$, corresponding to the $v \rightarrow 1$ case, the appropriate limit is $\omega_{+} \simeq q, q_{\max } \simeq E$, leading to a much more involved expression.

We can however obtain an analytical expression for the $v \rightarrow 1$ limit

$$
\begin{aligned}
\mathcal{I}_{2}^{(v=1)} & =\int_{0}^{\infty} d k\left[n_{+}(k)-n_{-}(k)\right] \frac{2}{3} k\left(3 E-5 k+2 \frac{k^{2}}{E}\right) \\
& =2 E T^{2}\left[L i_{2}\left(-e^{-\mu_{5} / T}\right)-L i_{2}\left(-e^{\mu_{5} / T}\right)\right]+\ldots,
\end{aligned}
$$

where the dots denote subleading terms in a $1 / E$ expansion, and $L i_{2}$ denotes the dilogarithm function. We note that for large $E$ this is a leading correction. If we further expand in the limit $\mu_{5} \ll T$, we obtain

$$
\mathcal{I}_{2}^{(v=1)} \simeq 2 E T \mu_{5} \log 2+\mathcal{O}\left(\frac{\mu_{5}}{T}\right)
$$

Putting together the two contributions in Eq. (13), we reach to the final hard contribution result, valid for $E \ll M^{2} / T$

$$
\begin{aligned}
\Delta_{\left(E \ll M^{2} / T\right)}^{\mathrm{hard}}= & \frac{e^{4} T^{2}}{12 \pi} \frac{\mu_{5}}{E}\left(1+\frac{\mu_{5}^{2}}{\pi^{2} T^{2}}\right)\left[\frac{3 v-5 v^{3}}{v^{3}\left(v^{2}-1\right)}\right. \\
& \left.-\frac{3}{2 v^{3}}\left(v^{2}-1\right) \log \frac{1+v}{1-v}\right]
\end{aligned}
$$

while for $v=1\left(E \gg M^{2} / T\right)$, in the limit $\mu_{5} \ll T$ the leading contribution is given by

$$
\Delta_{(v=1)}^{\mathrm{hard}} \approx \frac{e^{4}}{2 \pi^{3}} \mu_{5} T \log 2+\mathcal{O}\left(\frac{\mu_{5}}{T}, \frac{\mu_{5}}{E}\right),
$$

which becomes almost comparable with the leadingorder result for the collisional energy loss, which is 
$\mathcal{O}\left(e^{4} \log (e) T^{2}\right)$. It is also worth recalling that for $v=1$ the helicity of the fermion coincides with the chirality, and it is then a Lorentz invariant.

The other process which could contribute to the collisional energy loss is Compton scattering with medium photons. One can nevertheless see that, at this order of the calculation, all the relevant contributions which depend on the fermion helicity and the chiral imbalance of the system vanish, so that this process will not contribute to $\Delta$ at the same order as Eqs. (19) and (20).

\section{SOFT CONTRIBUTION TO THE COLLISIONAL ENERGY LOSS OF A MASSIVE FERMION IN THE CHIRAL PLASMA}

Let us now focus on the contribution to the energy loss coming from soft momentum photons, mainly focusing on contributions that depend on the helicity of the test fermion. The most convenient way to calculate these contributions is to start from the computation of the fermion damping rate [13]

$$
\gamma_{\lambda}=-\left.\frac{1}{2 E} \operatorname{Tr}\left[\mathcal{P}_{(\lambda, \mathbf{p})}(\not P+M) \operatorname{Im} \Sigma(P)\right]\right|_{p_{0}=E},
$$

where $\mathcal{P}_{(\lambda, \mathbf{p})}$ are the helicity projectors defined in Eq. (2), and the fermion self-energy $\Sigma(P)$ (Fig. 1 right) can be written in terms of four independent scalar functions $\Sigma_{s}^{\lambda}(P)$

$$
\Sigma(P)=\sum_{\lambda= \pm} \sum_{s= \pm} \mathcal{P}_{(\lambda, \mathbf{p})} \gamma_{0} \Lambda_{(s, \mathbf{p})} \Sigma_{s}^{\lambda}(P),
$$

where

$$
\Lambda_{( \pm, \mathbf{p})}=\frac{E \pm\left(\gamma_{0} \vec{\gamma} \cdot \mathbf{p}+\gamma_{0} M\right)}{2 E}
$$

are particle/antiparticle projectors. One can check that $\left[\mathcal{P}_{(\lambda, \mathbf{p})}, \Lambda_{( \pm, \mathbf{p})}\right]=0$.

The fermion self-energy can be computed e.g., using the imaginary time formalism, and then analytically continued to Minkowski space time. For a plasma without any chiral imbalance, the self-energy corrections for the two helicities turn out to be the same [14]. In the presence of a nonvanishing $\mu_{5}$ however, one can expect the damping rate to depend on the helicity of the fermion, only based on the global symmetries of the system, as parity is broken.

If we compute the fermion damping rate in the regime where the photon carries soft momentum, the photon in the one-loop diagram has to be resummed. If we use the hard thermal loop (HTL) resummed photon propagators, there is still no helicity dependence on the fermion damping rate. However, we find a helicity dependence if we improve the HTL resummation by considering $\mu_{5}$ corrections to the resummed photon propagators.
As in [8], we consider that in Coulomb gauge (we ignore gauge dependent pieces here, as they do not contribute to the imaginary part of the fermion self-energy) the photon propagator can be written as [15]

$$
D_{\mu \nu}(Q)=\delta_{\mu 0} \delta_{\nu 0} D_{L}(Q)+\sum_{h= \pm} \mathcal{P}_{\mu \nu}^{T, h} D_{T}^{h}(Q)
$$

where $h= \pm$ labels the two circular polarized transverse photon states, left and right, and

$$
\mathcal{P}_{\mu \nu}^{T, h}=\frac{1}{2}\left(\delta^{i j}-\hat{q}^{i} \hat{q}^{j}-i h \epsilon^{i j k} \hat{q}^{k}\right) \delta_{\mu i} \delta_{\nu j} .
$$

The resummed longitudinal and transverse propagators read, with the usual prescription $\omega \rightarrow \omega \pm i \eta$ for retarded and advanced quantities, respectively,

$D_{L}(\omega, q)=\frac{1}{q^{2}+\Pi_{L}}, \quad D_{T}^{h}(\omega, q)=\frac{1}{\omega^{2}-q^{2}-\Pi_{T}-h \Pi_{P}}$,

where

$$
\begin{gathered}
\Pi_{L}(\omega, q)=m_{D}^{2}\left(1-\frac{\omega}{2 q} \ln \frac{\omega+q}{\omega-q}\right), \\
\Pi_{T}(\omega, q)=m_{D}^{2} \frac{\omega^{2}}{2 q^{2}}\left[1+\frac{1}{2}\left(\frac{q}{\omega}-\frac{\omega}{q}\right) \ln \frac{\omega+q}{\omega-q}\right]
\end{gathered}
$$

are the longitudinal/transverse part of the hard thermal/ dense loop photon polarization tensor [16], and $m_{D}^{2}=$ $e^{2}\left(\frac{T^{2}}{3}+\frac{\mu_{5}^{2}}{\pi^{2}}\right)$ is the Debye mass, while

$$
\Pi_{P}(Q)=-\frac{e^{2} \mu_{5}}{\pi^{2}} \frac{\omega^{2}-q^{2}}{q}\left[1-\frac{\omega}{2 q} \ln \frac{\omega+q}{\omega-q}\right]
$$

can be viewed as the anomalous hard dense loop contribution [17-19].

Please also note that by improving the HTL resummed photon propagators by adding the new anomalous contribution, means that we are including a correction of order $e$ to the standard HTL result, as it is a correction $\sim e^{2} \mu_{5} q_{\text {soft }} \sim e q_{\text {soft }}^{2}$, where $q_{\text {soft }}$ is a soft momentum, of order $e T$ or $e \mu_{5}$.

Let us point out here that by analyzing the poles of the transverse propagators, one finds a chiral plasma instability $[17,18]$. However, the timescales associated with the instability are relatively large $\left(t_{\text {ins }} \sim T^{2} / e^{4} \mu_{5}^{3}\right.$, for $\mu_{5} \sim T, t_{\text {ins }} \sim 1 / e^{4} \mu_{5}$ ), and the computation of the energy loss we carry out is valid for shorter timescales.

The spectral functions associated with the longitudinal and transverse gauge field modes are given by 


$$
\begin{gathered}
\rho_{L}(Q)=2 \operatorname{Im} D_{L}(\omega+i \eta, q), \\
\rho_{T}^{h}(Q)=2 \operatorname{Im} D_{T}^{h}(\omega+i \eta, q), \quad h= \pm,
\end{gathered}
$$

Using these resummed photon propagators, after some standard manipulations, one ends up with the following value of the interaction rate

respectively.

$$
\begin{aligned}
\Gamma_{\lambda}= & \frac{e^{2}}{2 E} \int \frac{d^{3} q}{(2 \pi)^{3}} \int d \omega\left[1+n_{B}(\omega)\right] \delta\left(E-E^{\prime}-\omega\right) \frac{1}{E^{\prime}}\left\{\left[E E^{\prime}+E^{2}-\mathbf{p} \cdot \mathbf{q}\right] \rho_{L}(Q)+\left[E E^{\prime}-M^{2}-(\mathbf{p} \cdot \hat{\mathbf{q}})^{2}+\mathbf{p} \cdot \mathbf{q}\right] \sum_{h= \pm} \rho_{T}^{h}(Q)\right. \\
& \left.-\lambda\left[\left(E E^{\prime}-M^{2}-p^{2}\right)(\hat{p} \cdot \hat{q})+p q\right] \sum_{h= \pm} h \rho_{T}^{h}(Q)\right\}
\end{aligned}
$$

where $E^{\prime}=\sqrt{(\mathbf{p}-\mathbf{q})^{2}+M^{2}}, n_{B}(\omega)=(\exp (\omega / \mathrm{T})-1)^{-1}$ is the bosonic occupation number and we assumed that $M, E^{\prime} \gg T$, which allows us to neglect the fermionic occupation numbers in our expression.

The above integral can be analyzed in an expansion on $1 / E$. Let us define the velocity vector as $\mathbf{v}=\mathbf{p} / E$ and expand

$$
E^{\prime}=\sqrt{(\mathbf{p}-\mathbf{q})^{2}+M^{2}} \simeq E-v q \cos \theta+\frac{q^{2}\left(1-v^{2} \cos ^{2} \theta\right)}{2 E}+\mathcal{O}\left(\frac{1}{E^{2}}\right)
$$

We can write

$$
\begin{aligned}
\Gamma_{\lambda}= & \frac{e^{2}}{4 \pi^{2} v} \int_{0}^{\infty} d q q \int_{-q v}^{q v} d \omega\left[1+n_{B}(\omega)\right]\left\{\left(1-\frac{\omega}{E}\right) \rho_{L}(\omega, q)+\frac{1}{2} \sum_{h= \pm} \rho_{T}^{h}(\omega, q)\left[\left(v^{2}-\frac{\omega^{2}}{q^{2}}\right)\left(1-\frac{\lambda h q}{v E}\right)-\frac{\omega}{E}\left(1-\frac{\omega^{2}}{q^{2}}\right)\right]\right\} \\
& -\frac{e^{2}\left(1-v^{2}\right)}{8 E \pi^{2} v} \int_{0}^{\infty} d q q^{3} \rho_{L}(q v, q),
\end{aligned}
$$

which in the limit of a massless fermion $v \rightarrow 1$ reduces to the expressions found in [8,20,21], as expected.

When $\mu_{5}=0$ our result agrees with that of Refs. $[9,10]$ up to order $1 / E$ (after taking into account a different factor of $2 \pi$ in the convention for the definition of the photon spectral functions). At order $1 / E$ we find the new contributions that only appear in the presence of chiral imbalance (as otherwise $\rho_{T}^{+}=\rho_{T}^{-}$and they would cancel when summing over polarizations), which depend on both the helicity of the fermion and the circular polarization of the photon.

The energy loss can again be obtained from the damping by multiplying by $\omega / v$ [9]. We have

$$
\begin{aligned}
-\left.\frac{d E_{\lambda}}{d x}\right|_{\text {soft }}= & e^{2} \int \frac{d^{3} q}{(2 \pi)^{3}} \int d \omega\left[1+n_{B}(\omega)\right] \delta\left(E-E^{\prime}-\omega\right) \frac{1}{2 E E^{\prime}} \frac{\omega}{v} \\
& \times\left\{\left[E E^{\prime}+E_{p}^{2}-\mathbf{p} \cdot \mathbf{q}\right] \rho_{L}(Q)+\left[E E^{\prime}-M^{2}-(\mathbf{p} \cdot \hat{\mathbf{q}})^{2}+\mathbf{p} \cdot \mathbf{q}\right] \sum_{h= \pm} \rho_{T}^{h}(Q)\right. \\
& \left.-\lambda\left[\left(E E^{\prime}-M^{2}-p^{2}\right)(\hat{p} \cdot \hat{q})+p q\right] \sum_{h= \pm} h \rho_{T}^{h}(Q)\right\}
\end{aligned}
$$

Focusing again on the difference between the energy loss of two particles with opposite helicities,

$$
\left.\Delta^{\mathrm{soft}} \equiv\left(-\frac{d E_{\lambda=+}}{d x}\right)\right|_{\mathrm{soft}}-\left.\left(-\frac{d E_{\lambda=-}}{d x}\right)\right|_{\mathrm{soft}}
$$

we get

$$
\Delta^{\mathrm{soft}}=\frac{e^{2}}{4 \pi^{2} v^{2}} \int_{0}^{\infty} d q q^{2} \int_{-v}^{v} d x(q x)\left[1+n_{B}(q x)\right] \frac{1}{2} \sum_{h= \pm} \rho_{T}^{h}(q x, q)\left(v^{2}-x^{2}\right)\left(-2 \frac{h q}{v E}\right)
$$


We can plug in the explicit value of the transverse spectral functions. One has [8]

$$
\begin{aligned}
\frac{\rho_{T, \mathrm{cut}}^{h}}{2 \pi}(\omega, q) & =\frac{M_{h}^{2} \frac{x}{1-x^{2}} \Theta\left(1-x^{2}\right)}{\left[2 q^{2}+\frac{m_{D}^{2}}{1-x^{2}}-M_{h}^{2} Q_{1}(x)\right]^{2}+\frac{M_{h}^{4} \pi^{2} x^{2}}{4}}, \\
x & =\omega / q,
\end{aligned}
$$

with $M_{h}^{2}=m_{D}^{2}-\frac{e^{2} \mu_{5}}{2 \pi^{2}} h q$,

$$
Q_{1}(x)=1-\frac{x}{2} \ln \left|\frac{1+x}{1-x}\right|,
$$

and we note that we can substitute $1+n_{B}(q x) \rightarrow \frac{1}{2}$ due to the symmetries of the integrand in the domain of integration.

At this point one could worry whether there could be problems of nonintegrability in the computation due to the chiral plasma instability. However, exactly as it occurs in the computation of the energy loss in anisotropic QED and QCD plasmas [22,23], which exhibit the well-known Weibel instabilities, we find that the poles associated with the unstable modes are dynamically shielded. The computation of other quantities such as the momentum broadening would however be more problematic, as there the unstable modes are not shielded [24,25], and would be drastically affected by the instability.

One can easily recognize that the resulting integrals contain both linear and quadratic ultraviolet divergencies, as opposed to the $\mu_{5}=0$ case, where the leading result in the soft region contains only a logarithmic ultraviolet divergence. In this case, it turns out to be very convenient to use dimensional regularization. The linear divergence is then set to zero in DR, and only yields a finite result proportional to $e^{2} m_{D}^{2} e^{2} \mu_{5} / E$ (see Appendix B). The dominant term of $\Delta^{\text {soft }}$ is then provided by the piece that is quadratically divergent: in $d=3+2 \epsilon$ spatial dimensions

$\Delta^{\mathrm{soft}} \approx \frac{e^{2}}{8 \pi v^{3}} \frac{e^{2} \mu_{5}}{E} \int_{-v}^{v} d x x^{2}\left(1-\frac{x^{2}}{v^{2}}\right)^{(d-3) / 2} \frac{v^{2}-x^{2}}{1-x^{2}} \sum_{h= \pm} \mathcal{I}_{q}^{h}(x)$,

with

$\mathcal{I}_{q}^{h}(x)=\nu^{3-d} F(d) \int_{0}^{\infty} d q \frac{q^{d+2}}{\left[2 q^{2}+\frac{m_{D}^{2}}{1-x^{2}}-M_{h}^{2} Q_{1}(x)\right]^{2}+\frac{M_{h}^{4} \pi^{2} x^{2}}{4}}$,

where $\nu$ is the DR scale, and $F(d)$ is a normalization factor in $d$ dimension, see Eq. (A2). A closed analytical expression for the above integral can be obtained if we neglect the $e^{2} \mu_{5}$ pieces in the denominator, giving (see Appendix B)

$$
\mathcal{I}_{q}^{h}(x) \approx \frac{m_{D}^{2}}{4 \pi^{2}} A(x)\left(\frac{1}{\epsilon}+\log \frac{\nu^{2}}{m_{D}^{2}}+\gamma-\log 4 \pi\right),
$$

with $A(x)=\frac{1}{2}\left(\frac{1}{1-x^{2}}-Q_{1}(x)\right)$. Thus

$\Delta^{\mathrm{soft}} \approx \frac{e^{4} m_{D}^{2}}{2 v^{3}} \frac{\mu_{5}}{E}\left(\frac{1}{\epsilon}+\log \frac{\nu^{2}}{m_{D}^{2}}+\gamma-\log 4 \pi\right) f(v)$,

where

$$
f(v)=2 \int_{-v}^{v} d x x^{2} \frac{v^{2}-x^{2}}{1-x^{2}} A(x)
$$

is a positive function for $v \in[0,1]$.

The divergence of $\Delta^{\text {soft }}$ in the limit $\epsilon \rightarrow 0$ should be cancelled by a hard contribution, as it occurs with the leading logarithmic divergence (see Appendix A). This would require us to compute perturbative corrections to the leading term we computed in the previous section, to find a quadratic infrared divergent piece. Using DR one then expects to eliminate the pole $1 / \epsilon$ and the dependence on the scale $\nu$, resulting in a contribution $\Delta \propto e^{4} \log \left(e^{2}\right) m_{D}^{2} \frac{\mu_{5}}{E}$, which would be a correction to the leading result computed in the previous section. It could also be possible that at this higher order of the computation the perturbative resummed theory breaks down. We defer the investigation of this issue to future projects, as this would be in any case a subleading correction.

\section{CONCLUSIONS}

We have computed the collisional energy loss of an energetic massive fermion crossing a chiral plasma with an imbalance of its left-handed and right-handed populations. In the presence of a chiral imbalance, the energetic fermion interacts differently with the left-handed and right-handed components of the plasma, generating new contributions to the energy loss. These contributions depend on the helicity of the fermion, and we isolated them by focusing on the difference between the energy loss of the two opposite helicities. Such contributions can only be due to parity breaking effects in the medium, and we single them out to study whether they can be used to quantify the chiral imbalance of the plasma.

We find that the leading contribution to these helicitydependent energy loss contributions comes from the exchange of hard photons with the medium constituents, and in some scenarios can become comparable to the leading-order result for a plasma without any chiral imbalance. More specifically, we find that for a very energetic fermion $(v \rightarrow 1)$ our correction is $\Delta \sim e^{4} T \mu_{5}$, compared to the known $\sim e^{4} \log \left(e^{2}\right) T^{2}$ result for a vanishing $\mu_{5}$ [9], whereas at smaller velocities we get a $\mu_{5} / E$ suppression compared to the leading result. 
On the other hand, when it comes to softer photon exchanges the chiral imbalance of the medium is felt via a different interaction with the right and left circular polarization components of the photon, which can be incorporated via an extension of the HTL resummation via the inclusion of an anomalous hard dense fermionic loop contribution proportional to the chiral imbalance of the medium $[17,18]$. This can be seen to be a subleading effect [8], perturbatively suppressed as compared with the contribution from the hard photon exchange.

Recall that chiral plasmas with an imbalance of rightand left-handed populations exhibit a chiral plasma instability [17,18], with a timescale $t_{\text {ins }} \sim T^{2} / e^{4} \mu_{5}^{3}$, and that our calculation for the fermion energy loss will be valid in a regime before the instability sets in. In practice, if we assume that the medium has a length of order $L$, we impose $L / v \ll t_{\text {ins }}$. The time scale should definitely be large for the QED plasma considered in this work.

While we have focused our computations to an electromagnetic plasma, it is easy to generalize them to QCD [26-29]. First, one has to take into account the proper color and flavor factors in the corresponding scattering matrix. Note that in QCD there is an additional diagram contributing to the hard sector of the energy loss, namely the collision of the energetic massive quark with the gluons of the medium, but it would not yield a helicity dependent piece in the energy loss, as one assumes that all gluons of different polarizations are equally thermally distributed. Then for a heavy quark traversing a chiral QCD plasma, composed by $N_{f}$ light quark flavors with a chiral chemical potential $\mu_{5}$, we can take our QED result, and simply replace the electromagnetic coupling constant by the strong coupling constant $e \rightarrow g$, and take into account a $2 N_{f} / 3$ global factor,

$$
\begin{aligned}
\Delta_{\left(E \ll M^{2} / T\right)}^{(\mathrm{QCD})}= & \frac{g^{4} T^{2} N_{f}}{18 \pi} \frac{\mu_{5}}{E}\left(1+\frac{\mu_{5}^{2}}{\pi^{2} T^{2}}\right)\left[\frac{3 v-5 v^{3}}{v^{3}\left(v^{2}-1\right)}\right. \\
& \left.-\frac{3}{2 v^{3}}\left(v^{2}-1\right) \log \frac{1+v}{1-v}\right]
\end{aligned}
$$

while for $v=1\left(E \gg M^{2} / T\right)$, in the limit $\mu_{5} \ll T$ the leading contribution is given by

$$
\Delta_{(v=1)}^{(\mathrm{QCD})} \approx \frac{g^{4} N_{f}}{3 \pi^{3}} \mu_{5} T \log 2+\mathcal{O}\left(\frac{\mu_{5}}{T}, \frac{\mu_{5}}{E}\right) .
$$

One should as well consider that the chiral plasma instability in the chromoelectromagnetic fields in this case should occur at $t_{\text {ins }} \sim T^{2} / g^{4} \mu_{5}^{3}$.

Our computations should be completed with the evaluation of a helicity dependence of the radiative energy loss. For very heavy fermions, it is known that collisional loss dominates over radiative loss. It might be particularly interesting to study the case of radiative energy loss for the $v \rightarrow 1$ case. This last computation would be needed to answer the question whether by analyzing the helicity dependence the energy loss of light energetic fermions one could get information of the chiral misbalance produced, for example, in heavy ion collisions.

\section{ACKNOWLEDGMENTS}

We thank J. Soto for instructive conversations on dimensional regularization, M. Carrington and D. d'Enterria for general discussions, and J. M. Torres-Rincón for a critical reading of the manuscript. We have been supported by MINECO (Spain) under the Projects No. FPA2016-81114$\mathrm{P}$ and PID2019-110165 GB-I00 (MCI/AEI/FEDER, UE), as well as by the Project No. 2017-SGR-929 (Catalonia). S. C. has also been supported by the MINECO (Spain) under the Projects No. FPA2016-76005-C2-1-P and PID2019-105614 GB-C21, and by the 2017-SGR-929 grant (Catalonia). This work was also supported by the COST Action CA15213 THOR.

\section{APPENDIX A: COLLISIONAL ENERGY LOSS IN A SYMMETRIC PLASMA USING DIMENSIONAL REGULARIZATION}

Most calculations of the energy loss of a fermion in a plasma (see e.g., Refs. $[9,10]$ ) have been performed by regularizing the intermediate results with a sharp cutoff separating the soft and hard pieces of the computation. The two results are then matched and the final cutoff dependence disappears.

The use of a sharp cutoff however has some disadvantages. It does not preserve gauge invariance, and makes the cancellation of powerlike divergencies more subtle, should they appear. It may thus be preferable to use a more refined method.

In this Appendix, we apply dimensional regularization to derive the leading-order result for the energy loss of a massive fermion in a plasma.

Working in $d=3+2 \epsilon$ spatial dimensions, our integrals are modified as

$$
\int \frac{d^{3} q}{(2 \pi)^{3}} \rightarrow \int \frac{d^{d} q}{(2 \pi)^{d}}=F(d) \int_{0}^{\infty} d q q^{d-1} \int_{-1}^{1} d \cos \theta \sin ^{d-3} \theta,
$$

with

$$
F(d)=\frac{4}{(4 \pi)^{\frac{d+1}{2}} \Gamma\left(\frac{d-1}{2}\right)}=\frac{1}{4 \pi^{2}}+\mathcal{O}(\epsilon)
$$

where $\theta$ parametrizes an angle with respect to an external vector, and $\Gamma(z)$ stands for the Gamma function. Furthermore, in $d$ dimensions one has to change the 
coupling constant as $e^{2} \rightarrow e^{2} \nu^{3-d}$, where $\nu$ is an auxiliary scale introduced by DR.

In a similar spirit to previous calculations, we then compute separately the contributions at the soft and hard scales, which will exhibit ultraviolet and infrared divergencies, respectively, appearing as simple poles $1 / \epsilon_{\mathrm{UV}}$ and $1 / \epsilon_{\mathrm{IR}}$. The two results are then matched at the scale $\nu$, with the identification $\epsilon_{\mathrm{IR}}=\epsilon_{\mathrm{UV}}$, and the poles as well as all dependence on the scale $\nu$ cancel out.

Dimensional regularization has several advantages over cutoff regularization (see [30] for a general discussion on DR and effective field theories, or [31-33] for explicit applications for thermal plasmas). In DR scaleless integrals vanish, and there are no power divergences. Evaluating integrals using DR is basically the same as evaluating integrals using the method of residues [30], the result is given in terms of the residues of these poles, which only depend on the physical scales of the theory.

As we will show in our explicit computations of the energy loss in the soft sector, even if the integrals are carried out for all momenta, the integral is dominated by $e T$, the soft scale associated with the Debye mass, as it is the only scale in the corresponding integral. In an analogous way, in the hard sector, the integrals are dominated by contributions at the scale given by the temperature $T$, as can be seen by the presence of the fermionic occupation numbers.

Let us illustrate how this works by repeating the computation [9] using DR.

\section{Soft contribution}

First we compute the soft contribution to the energy loss using the HTL effective theory. Within dimensionally regularized resummed effective field theory the computation will have UV divergences, which appear as simple poles in our result.
Generalizing the result of [9] to $d$ dimensions, we have

$$
\begin{aligned}
-\left.\frac{d E}{d x}\right|_{\mathrm{soft}}= & \frac{e^{2} \nu^{3-d}}{v^{2}} F(d) \int_{0}^{\infty} d q q^{d-1} \int_{-v}^{v} d x\left(1-\frac{x^{2}}{v^{2}}\right) \\
& \times\left[1+n_{B}(q x)\right](q x)\left(\rho_{L}^{d}(q x, q) / 2\right. \\
& \left.+\left(v^{2}-x^{2}\right) \rho_{T}^{d}(q x, q)\right)
\end{aligned}
$$

where the factor $\left(1-\frac{x^{2}}{v^{2}}\right)^{(d-3) / 2}$ stems from the angular integral in $d$ dimensions, and $\rho_{L / T}^{d}$ are the HTL spectral functions, which can be defined in Coulomb gauge as in the $d=3$ case starting from the imaginary part of the retarded propagators:

$$
\rho_{L / T}^{d}\left(q_{0}, q\right)=2 \operatorname{Im} D_{L / T}^{d}\left(q_{0}+i \epsilon, q\right)
$$

One can substitute $1+n_{B}(q x) \rightarrow \frac{1}{2}$ due to the symmetries of the integrand in the domain of integration in Eq. (A3). Then the only physical scale that appears in Eq. (A3) is the Debye mass.

Expressions of the HTL in $d$ dimensions can be found in $[34,35]$. The result is finite and does not present any sort of divergencies, as the scaleless $T=0$ integrals are zero in DR. One thus typically computes the HTL diagrams setting $\epsilon=0$ (i.e., $d=3$ ). However, since in our calculation they are multiplied by $1 / \epsilon$ poles, one should keep pieces up to order $\epsilon$, which can be seen as corrections to the Debye mass as well as the Landau damping pieces, as they can give rise to finite pieces in the final result. In the following, we will mainly focus on the form of the pole obtained within the DR calculation, so we will not derive these explicitly.

Performing the $q$ integral (see Appendix B for the explicit results), one finds, in the limit $\epsilon \rightarrow 0$,

$$
-\left.\frac{d E}{d x}\right|_{\text {soft }}=-\frac{e^{2} m_{D(3+2 \epsilon)}^{2}}{16 \pi}\left\{\left(\frac{1}{v}-\frac{1-v^{2}}{2 v^{2}} \ln \frac{1+v}{1-v}\right)\left(\frac{1}{\epsilon}-\ln \frac{\bar{\nu}^{2}}{m_{D}^{2}}\right)+A(v)+A^{\mathrm{extra}}(v)\right\},
$$

where $\bar{\nu}^{2}=\nu^{2}\left(4 \pi e^{-\gamma}\right), m_{D(3+2 \epsilon)}^{2}$ denotes the Debye mass in $d=3+2 \epsilon$ dimensions keeping pieces up to $\mathcal{O}(\epsilon)$, which is given by (for simplicity we restrict ourselves to $\mu=0$ )

$m_{D(3+2 \epsilon)}^{2}=16 e^{2} F(3+2 \epsilon) \nu^{-2 \epsilon} \int_{0}^{\infty} d k k^{1+2 \epsilon} n_{F}(k)=m_{D}^{2}\left[1+\epsilon\left(2-\gamma_{E}+2 \frac{\zeta^{\prime}(2)}{\zeta(2)}-\log \frac{\pi \nu^{2}}{T^{2}}\right)\right]=m_{D}^{2}+\epsilon\left(\delta m_{D}^{2}\right)$,

where $n_{F}$ is the fermionic occupation number, $\zeta$ denotes the Riemann zeta function, and $\zeta^{\prime}$ its derivative.

The finite pieces are given by

$$
\begin{aligned}
A(v) v^{2}= & \int_{-v}^{v} d x x^{2} \ln \left(1-\frac{x^{2}}{v^{2}}\right)\left(1+\frac{1}{2} \frac{v^{2}-x^{2}}{1-x^{2}}\right)+\frac{1}{2} \int_{-v}^{v} d x x^{2}\left(\ln \left(Q_{1}^{2}(x)+\frac{\pi^{2} x^{2}}{4}\right)+\frac{1}{2} \frac{v^{2}-x^{2}}{1-x^{2}} \ln \left(Q_{2}^{2}(x)+\frac{\pi^{2} x^{2}}{16}\right)\right) \\
& +\int_{-v}^{v} d x x^{2}\left(\frac{2 Q_{1}(x)}{\pi x} \arccos \frac{\pi x}{2 Q_{1}(x)}+\frac{1}{2} \frac{v^{2}-x^{2}}{1-x^{2}} \frac{Q_{2}(x)}{\pi x} \arccos \frac{\pi x}{Q_{2}(x)}\right),
\end{aligned}
$$


which matches the finite pieces of the soft contribution of the energy loss computed with a cutoff of Ref. [9], and $A^{\text {extra }}$ are additional pieces arising from considering HTLs in $d=3+2 \epsilon$ dimensions.

Focusing on the pole, we get

$$
-\left.\frac{d E}{d x}\right|_{\mathrm{soft}} ^{\mathrm{pole}}=-\frac{e^{2} m_{D}^{2}}{16 \pi}\left(\frac{1}{v}-\frac{1-v^{2}}{2 v^{2}} \ln \frac{1+v}{1-v}\right) \frac{1}{\epsilon}
$$

and we need to check if it cancels with the hard part.

\section{Hard contribution}

The computation above has to be matched with that of the tree-level scattering in QED, which we also carry out in $d=3+2 \epsilon$ spatial dimensions. This one contains IR divergencies, which are again regulated with DR.

Restricting ourselves to the leading terms in the $1 / E$ expansion, and considering $\mu=0$ for simplicity, we have [9]

$$
\begin{aligned}
-\left.\frac{d E}{d x}\right|_{\text {hard }}= & \frac{4 \pi e^{4} \nu^{6-2 d}}{v} \int \frac{d^{d} k}{(2 \pi)^{d}} n_{F}(k) \int \frac{d^{d} q}{(2 \pi)^{d}} \int d \omega \delta(\omega+k-|\mathbf{k}+\mathbf{q}|) \delta(\omega-\mathbf{v} \cdot \mathbf{q}) \\
& \times \frac{\omega}{Q^{4}} \frac{1}{k|\mathbf{k}+\mathbf{q}|}\left(2(k-\mathbf{v} \cdot \mathbf{k})^{2}+\frac{1-v^{2}}{2} Q^{2}\right),
\end{aligned}
$$

where $n_{F}$ is the fermionic occupation number. At this point we can perform an average of the above expression on the directions of $\mathbf{v}$.

The angular integral will give us new integration boundaries, as well as an additional factor from the integration measure in $d$ dimensions. We get to an expression which can be split into two integrals over different boundaries for $q$ and $\omega$ :

$$
\begin{aligned}
-\left.\frac{d E}{d x}\right|_{\text {hard }}= & \frac{2 e^{4} \nu^{6-2 d}}{v^{2}} \frac{2 \pi^{d / 2}}{\sqrt{\pi} \Gamma\left(\frac{d-1}{2}\right)} \frac{F(d)}{(2 \pi)^{d-1}} \int_{0}^{\infty} d k k^{d-3} n_{F}(k)\left\{\int_{0}^{2 k /(1+v)} d q q^{d-5} \int_{-v q}^{v q} d \omega \omega\right. \\
& \left.+\int_{2 k /(1+v)}^{2 k /(1-v)} d q q^{d-5} \int_{q-2 k}^{v q} d \omega \omega\right\}\left(\frac{1-v^{2}}{2} \frac{q^{2}}{Q^{2}}+\frac{3 \omega^{2}-v^{2} q^{2}}{4 q^{2}}+\frac{3\left(\omega k+k^{2}\right)}{q^{2}}\right. \\
& \left.-\left(v^{2}-1\right) \frac{\omega k+k^{2}}{Q^{2}}\right)\left(1-\frac{\omega^{2}}{v^{2} q^{2}}\right)^{(d-3) / 2}\left[1-\left(\frac{Q^{2}}{2 k q}+\frac{\omega}{q}\right)^{2}\right]^{(d-3) / 2} \equiv I_{1}+I_{2} .
\end{aligned}
$$

Since our main focus in this section is to discuss the implementation of dimensional regularization, we focus on the possibly diverging pieces, which are restricted to the first $q$ integral above, which is IR divergent. The remaining pieces are the same as those obtained in $[9,10]$.

Focusing on the first integral of Eq. (A10) and setting $d=3+2 \epsilon$ we have

$$
\begin{aligned}
I_{1}= & \frac{e^{4} \nu^{-4 \epsilon}}{v^{2} \pi^{2}} \frac{1}{(4 \pi)^{1+2 \epsilon}} \frac{1}{(\Gamma(1+\epsilon))^{2}} \int_{0}^{\infty} d k k^{2 \epsilon} n_{F}(k) \int_{0}^{2 k /(1+v)} d q q^{-2+2 \epsilon} \int_{-v q}^{v q} d \omega \\
& \times\left[\frac{1-v^{2}}{2} \frac{q^{2}}{Q^{2}}+\frac{3 \omega^{2}-v^{2} q^{2}}{4 q^{2}}+\frac{3\left(\omega k+k^{2}\right)}{q^{2}}-\left(v^{2}-1\right) \frac{\omega k+k^{2}}{Q^{2}}\right]\left(1-\frac{\omega^{2}}{v^{2} q^{2}}\right)^{\epsilon}\left[1-\left(\frac{Q^{2}}{2 k q}+\frac{\omega}{q}\right)^{2}\right]^{\epsilon} .
\end{aligned}
$$

If we now restrict ourselves to extracting the pole and the dependence on the scale $\nu$, we can actually drop several finite pieces and work with

$$
I_{1}^{\mathrm{pole}}=\frac{e^{4} \nu^{-4 \epsilon}}{v^{2} \pi^{2}} \frac{1}{(4 \pi)} \int_{0}^{\infty} d k k^{2 \epsilon} n_{F}(k) \int_{0}^{2 k /(1+v)} d q q^{-2+2 \epsilon} \int_{-v q}^{v q} d \omega\left(\frac{3 \omega^{2} k}{q^{2}}-\left(v^{2}-1\right) \frac{\omega^{2} k}{Q^{2}}\right)
$$

where we exploited the symmetry of the integral to drop all odd terms in $\omega$. The $\omega$ integral can now be easily carried out, leading to 


$$
I_{1}^{\mathrm{pole}}=\frac{2 e^{4} \nu^{-4 \epsilon}}{\pi} F(3+2 \epsilon)\left(\frac{1}{v}+\frac{\left(v^{2}-1\right)}{2 v^{2}} \ln \frac{1+v}{1-v}\right) \int_{0}^{\infty} d k k^{1+2 \epsilon} n_{F}(k) \int_{0}^{2 k /(1+v)} d q q^{-1-2 \epsilon}
$$

Now we have to evaluate the $q$ integral. Due to the finite upper integration limit, this integral does not vanish in DR and gives

$$
\nu^{-2 \epsilon} \int_{0}^{2 k /(1+v)} d q q^{-1+2 \epsilon}=\frac{1}{2 \epsilon}\left(\frac{2 k}{\nu(1+v)}\right)^{2 \epsilon}=\frac{1}{2 \epsilon}+\log \frac{2 k}{\nu(1+v)}+\mathcal{O}(\epsilon)
$$

so we are left with

$$
\begin{aligned}
I_{1}^{\text {pole }} & =\frac{2 e^{4}}{\pi} F(3+2 \epsilon)\left(\frac{1}{v}+\frac{\left(v^{2}-1\right)}{2 v^{2}} \ln \frac{1+v}{1-v}\right) \nu^{-2 \epsilon} \int_{0}^{\infty} d k k^{1+2 \epsilon} n_{F}(k)\left[\frac{1}{2 \epsilon}+\log \frac{2 k}{\nu(1+v)}\right] \\
& =\frac{e^{2}}{16 \pi v}\left(1-\frac{1-v^{2}}{2 v} \ln \frac{1+v}{1-v}\right)\left[\frac{1}{\epsilon} m_{D(3+2 \epsilon)}^{2}+\frac{8 e^{2}}{\pi^{2}} \int_{0}^{\infty} d k k n_{F}(k) \log \frac{2 k}{\nu(1+v)}\right],
\end{aligned}
$$

where we recognized in the first row the same integral as Eq. (A6), giving the Debye mass in $d=3+2 \epsilon$ dimensions.

Restricting ourselves for simplicity to the case $\mu=0$, we get

$$
-\left.\frac{d E}{d x}\right|_{\text {hard }}=\frac{e^{4} T^{2}}{48 \pi}\left[\frac{1}{v}+\frac{v^{2}-1}{2 v^{2}} \ln \frac{1+v}{1-v}\right]\left[\frac{1}{\epsilon}+\delta m_{D}^{2}-\log \frac{\bar{\nu}^{2}}{T^{2}}+\ldots\right],
$$

where $\delta m_{D}^{2}$ was defined in Eq. (A6) and the dots denote additional finite pieces. We can see that the pole thus cancels out exactly with the one of the soft part [Eq. (A8)], and the $\nu$ dependence also drops when adding up the two contributions, which combine to the characteristic $\log \left(e^{2}\right)$ factor.

The generalization to finite chemical potential amounts to replacing $T^{2} \rightarrow T^{2}\left(1+\frac{3 \mu^{2}}{\pi^{2} T^{2}}\right)$ in the pole, while for the finite pieces one obtains significantly more involved expressions, originating from the evaluation of integrals like $\int_{0}^{\infty} d k k \log (k)\left[\left(n_{F}(k, \mu)+n_{F}(k,-\mu)\right]\right.$. Remarkably, these are the same integrals appearing in the calculation of finite pieces at finite chemical potential using a sharp cutoff [36].

\section{APPENDIX B: USEFUL INTEGRALS IN DIMENSIONAL REGULARIZATION}

We report in this Appendix some integrals we encounter during our calculations, which are regularized in $d=3+2 \epsilon$ dimensions using DR. Recall that DR sets powerlike divergencies to zero, keeping only results which depend on the physical scales of the system.

The logarithmic ultraviolet divergence encountered in the soft region of the calculation for the energy loss in a thermal plasma without chiral imbalance stems from the integral

$$
\begin{aligned}
\nu^{-2 \epsilon} & \int_{0}^{\infty} d q \frac{q^{3+2 \epsilon}}{\left(q^{2}+m_{D}^{2} a\right)^{2}+m_{D}^{4} b^{2}}=\frac{1}{2}\left(\frac{m_{D}}{\nu}\right)^{2 \epsilon} \int_{0}^{\infty} d z \frac{z^{1+\epsilon}}{(z+a)^{2}+b^{2}} \\
= & -\frac{1}{2}\left(\frac{1}{\epsilon}-\log \frac{\nu^{2}}{m_{D}^{2}}+\frac{1}{2} \log \left(a^{2}+b^{2}\right)+\frac{a}{b} \arccos \frac{a}{\sqrt{a^{2}+b^{2}}}\right)+\mathcal{O}(\epsilon),
\end{aligned}
$$

and shows up as a $1 / \epsilon$ pole, together with a logarithmic dependence on the scale $\nu$, which are finally cancelled by similar contributions in the hard sector, which appear there as infrared divergent integrals.

When evaluating the new contributions arising in a chiral plasma, we deal with a possibly linearly ultraviolet divergent piece. In $d=3+2 \epsilon$ dimensions the corresponding integral reads

$$
\nu^{-2 \epsilon} \int_{0}^{\infty} d q \frac{q^{4+2 \epsilon}}{\left(q^{2}+m_{D}^{2} a\right)^{2}+m_{D}^{4} b^{2}}=\frac{m_{D}}{2}\left(\frac{m_{D}}{\nu}\right)^{2 \epsilon} \int_{0}^{\infty} d z \frac{z^{3 / 2+\epsilon}}{(z+a)^{2}+b^{2}}=-\frac{m_{D} \pi}{2 \sqrt{2}} \frac{2 a+\sqrt{a^{2}+b^{2}}}{\left(a+\sqrt{a^{2}+b^{2}}\right)^{1 / 2}}+\mathcal{O}(\epsilon),
$$

which however turns out to be finite in DR. 
In the chiral plasma there is also a quadratic ultraviolet divergence. In $d=3+2 \epsilon$ dimensions one has to evaluate

$$
\begin{aligned}
\nu^{-2 \epsilon} \int_{0}^{\infty} d q \frac{q^{5+2 \epsilon}}{\left(q^{2}+m_{D}^{2} a\right)^{2}+m_{D}^{4} b^{2}} & =\frac{m_{D}^{2}}{2}\left(\frac{m_{D}}{\nu}\right)^{2 \epsilon} \int_{0}^{\infty} d z \frac{z^{2+\epsilon}}{(z+a)^{2}+b^{2}} \\
& =a m_{D}^{2}\left(\frac{1}{\epsilon}-\log \frac{\nu^{2}}{m_{D}^{2}}+\frac{1}{2}\left[\log \left(a^{2}+b^{2}\right)+\frac{a^{2}-b^{2}}{a b} \arctan \frac{b}{a}\right]\right)+\mathcal{O}(\epsilon),
\end{aligned}
$$

which results in the 1/ $\epsilon$ pole appearing in Eq. (43), and a logarithmic dependence on the scale $\nu$, which should be cancelled with a contribution of the hard sector, not evaluated in this manuscript.

[1] D. d'Enterria, Landolt-Bornstein 23, 471 (2010).

[2] J. Casalderrey-Solana and C. A. Salgado, Acta Phys. Polon. B 38, 3731 (2007).

[3] A. Majumder and M. Van Leeuwen, Prog. Part. Nucl. Phys. 66, 41 (2011).

[4] G. Y. Qin and X. N. Wang, Int. J. Mod. Phys. E 24, 1530014 (2015).

[5] D. E. Kharzeev, Prog. Part. Nucl. Phys. 75, 133 (2014).

[6] D. E. Kharzeev, J. Liao, S. A. Voloshin, and G. Wang, Prog. Part. Nucl. Phys. 88, 1 (2016).

[7] X. G. Huang, Rep. Prog. Phys. 79, 076302 (2016).

[8] S. Carignano and C. Manuel, Phys. Rev. D 99, 096022 (2019).

[9] E. Braaten and M. H. Thoma, Phys. Rev. D 44, 1298 (1991).

[10] S. Peigne and A. Peshier, Phys. Rev. D 77, 014015 (2008).

[11] J. Hansen and K. Tuchin, arXiv:2012.06089.

[12] K. Tuchin, Phys. Lett. B 786, 249 (2018).

[13] H. A. Weldon, Phys. Rev. D 28, 2007 (1983).

[14] C. Manuel, Phys. Rev. D 62, 076009 (2000).

[15] J. F. Nieves and P. B. Pal, Phys. Rev. D 39, 652 (1989); 40, 2148(E) (1989).

[16] M. Le Bellac, Thermal Field Theory (Cambridge University Press, Cambridge, England, 1996).

[17] M. Laine, J. High Energy Phys. 10 (2005) 056.

[18] Y. Akamatsu and N. Yamamoto, Phys. Rev. Lett. 111, 052002 (2013).

[19] C. Manuel and J. M. Torres-Rincon, Phys. Rev. D 89, 096002 (2014).
[20] S. Carignano, C. Manuel, and J. M. Torres-Rincon, Phys. Rev. D 102, 016003 (2020).

[21] S. Carignano, C. Manuel, and J. M. Torres-Rincon, Phys. Rev. D 98, 076005 (2018).

[22] P. Romatschke and M. Strickland, Phys. Rev. D 69, 065005 (2004).

[23] P. Romatschke and M. Strickland, Phys. Rev. D 71, 125008 (2005).

[24] P. Romatschke, Phys. Rev. C 75, 014901 (2007).

[25] R. Baier and Y. Mehtar-Tani, Phys. Rev. C 78, 064906 (2008).

[26] E. Braaten and M. H. Thoma, Phys. Rev. D 44, R2625 (1991).

[27] A. Peshier, Phys. Rev. Lett. 97, 212301 (2006).

[28] S. Peigne and A. Peshier, Phys. Rev. D 77, 114017 (2008).

[29] S. Peigne and A. V. Smilga, Phys. Usp. 52, 659 (2009).

[30] A. V. Manohar, Les Houches Lect. Notes 108 (2020) 10.1093/oso/9780198855743.003.0002.

[31] M. A. Escobedo and J. Soto, Phys. Rev. A 78, 032520 (2008).

[32] M. A. Escobedo and J. Soto, Phys. Rev. A 82, 042506 (2010).

[33] M. A. Escobedo, J. Soto, and M. Mannarelli, Phys. Rev. D 84, 016008 (2011).

[34] S. Carignano, C. Manuel, and J. Soto, Phys. Lett. B 780, 308 (2018).

[35] S. Carignano, M. E. Carrington, and J. Soto, Phys. Lett. B 801, 135193 (2020).

[36] H. Vija and M. H. Thoma, Phys. Lett. B 342, 212 (1995). 